\title{
ATIVIDADE ANTIFÚNGICA IN SILICO E IN VITRO DO MONOTERPENO CITRAL
}

\author{
Camilla Pinheiro de Menezes CALDAS ${ }^{1}$
}

Janiere Pereira de SOUSA ${ }^{2}$

Ana Luíza Alves de Lima PÉREZ

Jéssyca Marina Carneiro Gomes dos SANTOS ${ }^{4}$

Heloísa Mara Batista Fernandes de OLIVEIRA ${ }^{5}$

Abrahão Alves de OLIVEIRA FILHO ${ }^{6}$

Edeltrudes de Oliveira LIMA ${ }^{7}$

1. Doutoranda em Produtos Naturais e Sintéticos Bioativos pela Universidade Federal da Paraíba, João Pessoa, Paraíba, Brasil. E-mail: camilla.farmaufpb@gmail.com

2. Doutora em Produtos Naturais e Sintéticos Bioativos pela Universidade Federal da Paraíba, João Pessoa, Paraíba, Brasil. E-mail: janiereps@yahoo.com.br

3. Mestre em Odontologia pela Universidade Federal da Paraíba, João Pessoa, Paraíba, Brasil. E-mail: analuiza_perez@yahoo.com.br

4. Graduada em Farmácia pela Universidade Federal da Paraíba, João Pessoa, Paraíba, Brasil. E-mail: jessygomes_13@hotmail.com

5. Doutora em Produtos Naturais e Sintéticos Bioativos pela Universidade Federal da Paraíba e FarmacêuticaBioquímica do Hospital Ana Bezerra-UFRN. E-mail: heloisambf@gmail.com

6. Professor da Universidade Federal de Campina Grande, Doutor em Farmacologia. E-mail: abrahão.farm@gmail.com

7. Orientadora e Professora da Universidade Federal da Paraíba, João Pessoa, Paraíba, Brasil. E-mail:

edelolima@yahoo.com.br

Recebido em: 11/07/2016 - Aprovado em: 16/10/2016 - Disponibilizado em: 18/12/2016

\section{RESUMO}

Introdução: as pesquisas com monoterpenos têm aumentado com a necessidade de novas ferramentas terapêuticas para o combate das infecções fúngicas oportunistas, como as causadas pelos fungos dematiáceos pertencentes ao gênero Cladosporium. Objetivo: avaliar o potencial antifúngico do monoterpeno citral. Métodos: a determinação da CIM (Concentração inibitória mínima) das substâncias, foi realizada através da técnica da microdiluição em caldo. Utilizouse duas cepas de $C$. oxysporum e duas cepas de $C$. sphaerospermum. Todas as cepas de micro-organismos utilizados neste estudo fazem parte da Micoteca URM do Departamento de Micologia, Centro de Ciências Biológicas, Universidade Federal de Pernambuco. Foi realizado controle de viabilidade das cepas ensaiadas, e também controle de sensibilidade destas cepas frente à ação de antimicrobianos considerados padrões na utilização clínica. Resultados: pode-se observar que todos os compostos avaliados apresentaram uma $\mathrm{CIM}_{50}$ (Concentração Inibitória Mínima para $50 \%$ das cepas testadas) de $128 \mu \mathrm{g} / \mathrm{mL}$ para as cepas fúngicas testadas. Conclusão: conclui-se que o monoterpneo citral pode se tornar uma alternativa para o tratamento de infecções fúngicas causadas por cepas de Cladosporium.

Palavras-chave: antifúngico; monoterpeno; citral; Cladosporium oxysporum; Cladosporium sphaerospermum.

\section{ANTIFUNGAL ACTIVITY IN SILICO AND IN VITRO OF CITRAL MONOTERPENE}

\begin{abstract}
Introduction: research with monoterpenes have increased the need for new therapeutic tools to combat opportunistic fungal infections such as those caused by fungi dematiaceous belonging to the Cladosporium genus. Objective: To
\end{abstract}


evaluate the antifungal potential of citral monoterpene. Methods: The determination of the MIC (minimum inhibitory concentration) of the substance was performed using microdilution broth technique. We used two strains of $C$. oxysporum and two strains of $C$. sphaerospermum. All the strains of microorganisms used in this study are part of URM Culture Collection of the Department of Mycology, Center of Biological Sciences, Federal University of Pernambuco. Control was carried viability of the tested strains, and also control sensitivity of these strains opposite antimicrobial action patterns considered in the clinical use. Results: It can be seen that all the compounds evaluated showed a $\mathrm{MIC}_{50}$ (Minimum Inhibitory Concentration for $50 \%$ of tested strains) to $128 \mu \mathrm{g} / \mathrm{mL}$ for the tested fungal strains. Conclusion: It was concluded that citral monoterpene can become an alternative for the treatment of fungal infections caused by strains of Cladosporium.

Keywords: antifungal; monoterpene; citral; Cladosporium oxysporum, Cladosporium sphaerospermum.

\section{INTRODUÇÃO}

Os produtos químicos produzidos pelos vegetais podem ser divididos em dois grandes grupos. Os primeiros, denominados metabólitos primários ou macromoléculas, são essenciais a todos os seres vivos, e o segundo grupo de compostos químicos - os metabólitos secundários ou micromoléculas que geralmente apresentam estrutura complexa, baixo peso molecular, marcantes atividades biológicas e, diferentemente daqueles do metabolismo primário, são encontrados em concentrações relativamente baixas e em determinados grupos de plantas (POSER, MENTZ, 2004).

Os metabólitos secundários apresentam várias atividades biológicas. Muitos são de importância comercial tanto na área farmacêutica quanto nas áreas alimentar, agronômica e de perfumaria, entre outras. Entre os metabólitos secundários, os principais grupos de compostos encontrados com atividade biológica são os alcaloides, flavonoides, cumarinas, taninos, quinonas e óleos essenciais (PEREIRA, 2006). Quimicamente, a maioria dos óleos essenciais é constituída de derivados fenilpropanóides ou de terpenóides, sendo os monoterpenos (cerca de $90 \%$ dos óleos voláteis) e os sesquiterpenos os mais frequentes (SIMÕES, SPITZER, 2004).

Dentre os monoterpenos relatados na literatura destaca-se o citral, um monoterpeno acíclico natural, citral está presente no óleo essencial de várias espécies de plantas, incluindo limão e laranja (FISHER, PHILLIPS, 2008). E é o principal componente do óleo de Melissa officinalis pertencente à família Lamiaceae (SADDIQ, KHAYYAT, 2010). O citral é um monoterpeno com conhecidas propriedades farmacológicas, como: anti-tumoral ( FARAH et al., 2010; CHAOUKI et al., 2009; XIA et al., 2013; DUDAI et al., 2005), broncodilatador (MANGPRAYOOL, KUPITTAYANANT, CHUDAPONGSE, 2013), inseticida (ABRAMSON, ALDAMA, SULBARAN, 2007) e antimicrobiana (SOMOLINOS et al., 2009; BELDAGALBIS et al., 2013).

O gênero Cladosporium abrange muitas espécies de fungos contaminantes e oportunistas dematiáceos que são 
encontrados ubiquamente como saprófitas no solo e em materiais em decomposição (SCHUBERT, 2005).

As espécies $C$. cladosporioides, $C$. herbarum, C. oxysporum, C. carrionii e $C$. sphaerospermum têm sido observadas como responsáveis primárias por quadro de feohifomicoses superficiais (onicomicoses, ceratites, etc.), cromoblastomicoses e feohifomicoses profundas (meningites, quadro pulmonares, etc.) (DE HOOG et al., 2000; KWON-CHUNG, SCHWARTZ, RYBAK, 1975; NAMRATHA et al., 2010; CORREIA et al., 2010).

Nesse contexto, este estudo teve como objetivo avaliar in silico e in vitro o potencial antifúngico do monoterpeno citral contra cepas de $C$. oxysporum e $C$. sphaerospermum.

\section{MATERIAL E MÉTODOS}

\section{LOCAL DA PESQUISA}

A pesquisa foi realizada no Laboratório de Micologia do Departamento de Ciências Farmacêuticas (DCF), Centro de Ciências da Saúde (CCS), Universidade Federal da Paraíba (UFPB), no período de maio de 2015 a junho de 2016.

\section{CEPAS FÚNGICAS}

Para realização dos ensaios de atividade antifúngica in vitro foram selecionadas 2 cepas de $C$. oxysporum (
URM 5412 e URM 6056) e 2 cepas de $C$. sphaerospermum (URM 5350 e URM 5455) pertencentes à coleção de culturas da Micoteca URM do Departamento de Micologia, Centro de Ciências Biológicas, Universidade Federal de Pernambuco. Todas as cepas foram mantidas em Ágar Sabouraud Dextrose - ASD inclinado (DIFCO Laboratories Ltda, USA) a temperatura ambiente $\left(28^{\circ} \mathrm{C}\right)$ e sob refrigeração $\left(4^{\circ} \mathrm{C}\right)$.

Foi utilizado um inóculo fúngico de aproximadamente $1-5$ x $10^{6} \mathrm{UFC} / \mathrm{mL}$ padronizado de acordo com a turbidez do tubo 0,5 da escala de McFarland (CLEELAND, $\quad$ SQUIRES, 1991; HADACEK, GREGER, 2000; CLSI, 2002).

\section{MEIOS DE CULTURA}

Foram utilizados os meios ágar Sabouraud dextrose (ASD) e caldo Sabouraud dextrose (CSD) obtidos da Difco Laboratories (Detroit, MI, EUA) e RPMI1640-L-glutamina (sem bicarbonato de sódio) da Sigma-Aldrich ${ }^{\circledR}$ (São Paulo, SP, Brasil), preparados conforme as instruções do fabricante.

\section{MONOTERPENO E ANTIFÚNGICOS}

$$
\text { O monoterpeno (citral) e os }
$$
antifúngicos (anfotericina B e voriconazol) foram adquiridos da Sigma-Aldrich ${ }^{\circledR}$. As emulsões, nas diferentes concentrações, foram preparadas no momento de execução dos ensaios, dissolvendo-os primeiramente 
em dimetilsulfóxido (DMSO) a $0,5 \%$ e Tween $80 \quad\left(\right.$ Sigma-Aldrich $\left.^{\circledR}\right)$ a $2 \%$, e utilizando água destilada estéril para alcançar a concentração desejada.

\section{PASS ON LINE}

Previsão do espectro de atividade para substâncias (PASS) online (http://www. way2drug.com/passonline) é um software destinado para avaliar o potencial biológico geral de uma molécula orgânica sobre o organismo humano. Este programa fornece previsões simultâneas de muitos tipos de atividades biológicas com base na estrutura dos compostos orgânicos. O espectro de atividade biológica de um composto químico é o conjunto de diferentes tipos de atividade biológica, que refletem os resultados de interação do composto com várias entidades biológicas. Pass online dá várias facetas da ação biológica de um composto, obtendo os índices Pa (probabilidade " de ser ativo") e Pi (probabilidade "de ser inativo") estimando a categorização de um composto potencial em ser pertencente à subclasse de compostos ativos ou inativos, respectivamente (SRINIVAS et al., 2014).

PASS oferece previsões com base na probabilidade de novos efeitos $\mathrm{e}$ mecanismos de ação com espectro de atividade requerido entre os compostos a partir de bases de dados internas, antigas e comerciais. Pass online prevê o espectro de atividade biológica para as impressões modificadas, com base em sua fórmula estrutural, juntamente com diferentes descritores como antifúngica, antiviral, anti-helmíntico, antiprotozoários, etc., por isso, é possível estimar se os novos compostos têm um efeito particular (SRINIVAS et al., 2014).

\section{DETERMINAÇÃO DA CONCENTRAÇÃO INIBITÓRIA MÍNIMA (CIM)}

A concentração inibitória mínima do citral, bem como dos antifúngicos anfotericina B e voriconazol foi determinada pela técnica da microdiluição em caldo CLEELAND, SQUIRES, 1991; HADACEK, GREGER, 2000; CLSI, 2002; Eloff, 1998). Foram utilizadas placas de 96 orifícios estéreis e com tampa. Em cada orifício da placa, foi adicionado $100 \mu \mathrm{L}$ de RPMI-1640 duplamente concentrado. Em seguida, 100 $\mu \mathrm{L}$ da emulsão dos produtos, também duplamente concentrado, foram dispensados nas cavidades da primeira linha da placa. E por meio de uma diluição seriada a uma razão de dois, foram obtidas concentrações de $1024 \mu \mathrm{g} / \mathrm{mL}$ até $0,5 \mu \mathrm{g} / \mathrm{mL}$, de modo que na primeira linha da placa se encontra a maior concentração e na última, a menor concentração. Por fim, foi adicionado $10 \mu \mathrm{L}$ do inóculo de aproximadamente 1-5 x $10^{6}$ UFC/mL das espécies nas cavidades, onde cada coluna da placa refere-se a uma cepa fúngica, especificamente. 
Paralelamente, foi realizado controle de viabilidade das cepas ensaiadas $(100 \mu \mathrm{L}$ do mesmo RPMI-1640 duplamente concentrado e $10 \mu \mathrm{L}$ do inóculo de cada cepa). E para verificar a ausência de interferência nos resultados pelos agentes emulsificantes utilizados na solubilização do monoterpeno, foi feito um controle no qual foi colocado nas cavidades $100 \mu \mathrm{L}$ do RPMI1640, DMSO (0,5\%), Tween $80(2 \%)$ e 10 $\mu \mathrm{L}$ da suspensão. Um controle de esterilidade do meio também foi realizado, colocando-se $100 \mu \mathrm{L}$ do RPMI-1640 em cavidades sem a suspensão fúngica.

As placas foram assepticamente fechadas e incubadas a $28{ }^{\circ} \mathrm{C}$ por 5-7 dias para a realização da leitura. A CIM foi definida como a menor concentração capaz de inibir o crescimento fúngico visualmente verificado nos orifícios quando comparado com o crescimento controle. Os ensaios foram realizados em triplicata e a média geométrica foi calculada.

\section{RESULTADOS}

A análise dos valores de "Pa" no estudo in silico do citral revela que este monoterpeno apresenta uma probabilidade de atividades farmacológicas anti-infecciosa, em especial, atividade antifúngica $(\mathrm{Pa}=0,429$ e $\mathrm{Pi}=0,044)($ Tabela 1).
Tabela 1- Valores de Pa e Pi para o monoterpeno citral.

\begin{tabular}{ccc}
\hline $\begin{array}{c}\text { Valor de } \\
\mathbf{P a}\end{array}$ & $\begin{array}{c}\text { Valor de } \\
\mathbf{P i}\end{array}$ & Atividades farmacológicas \\
\hline 0,395 & 0,005 & Antiviral (CMV) \\
0,340 & 0,066 & Antiviral (Herpes) \\
0,281 & 0,103 & Antiviral (Influenza) \\
0,363 & 0,143 & Antiviral (Picornavirus) \\
0,228 & 0,120 & Antiviral (Poxvirus) \\
0,735 & 0,002 & Antiviral (Rhinovirus) \\
0,233 & 0,081 & Antiprotozoal \\
0,235 & 0,129 & Antiprotozoal (Coccidial) \\
0,335 & 0,079 & Antiprotozoal (Leishmania) \\
0,230 & 0,035 & Antiprotozoal (Plasmodium) \\
0,194 & 0,068 & Antiprotozoal (Toxoplasma) \\
0,353 & 0,060 & Antiprotozoal \\
0,421 & 0,014 & (Trypanosoma) \\
0,136 & 0,126 & Antihelmíntico \\
0,260 & 0,150 & Antihelmíntico (Nematodes) \\
0,429 & 0,044 & Antifúngico \\
0,377 & 0,036 & Antibacteriano \\
\hline Fontico (Fasciola)
\end{tabular}

Fonte: SANTOS, JMCG

Observando os valores da CIM (Concentração Inibitória Mínima) do citral para as cepas de $C$. oxysporum e $C$. sphaerospermum pode-se perceber que o composto testado apresenta uma CIM de 128 $\mu \mathrm{g} / \mathrm{mL}$ para três cepas avaliadas ( $C$. oxysporum URM 6056 e URM 5412, C. sphaerospermum URM 5350) e de 256 $\mu \mathrm{g} / \mathrm{mL}$ para uma das cepas testadas ( $C$. sphaerospermum URM 5455). Além disso, observa-se que os antifúngicos padrões, voriconazol e anfotericina $\mathrm{B}$, foram também capazes de inibir o crescimento fúngico, exceto para a cepa C. oxysporum URM 6056, que foi resistente a maior concentração testada $(1024 \mu \mathrm{g} / \mathrm{mL})$ dos dois antifúngicos padrão (Tabela 2). 
Tabela 2 - Resultados da CIM do citral, voriconazol e anfotericina B contra $C$. oxysporum e $C$. sphaerospermum

\begin{tabular}{|c|c|c|c|c|c|}
\hline \multirow{2}{*}{$\begin{array}{c}\text { Cepas de } \\
\text { Cladosporium }\end{array}$} & $\begin{array}{c}\text { Citral } \\
(\mu \mathrm{g} / \mathrm{mL})\end{array}$ & $\begin{array}{c}\text { Voriconazol } \\
(\mu \mathrm{g} / \mathrm{mL})\end{array}$ & $\begin{array}{l}\text { Anfotericina } \\
\mathrm{B}(\mu \mathrm{g} / \mathrm{mL})\end{array}$ & $* \mathrm{C}$ & \multirow{2}{*}{$\begin{array}{l}\text { Estes dados computacionais, por sua } \\
\text { foram confirmados com o estudo in vitro }\end{array}$} \\
\hline & CIM & CIM & CIM & & \\
\hline $\begin{array}{l}\text { C. oxysporum } \\
\text { URM } 6056\end{array}$ & 128 & $>1024$ & $>1024$ & & diferentes cepas de $C$. oxysporum \\
\hline $\begin{array}{l}\text { C. oxysporum } \\
\text { URM } 5412 \\
\end{array}$ & 128 & 8 & 16 & & sphaerospermum, contra as quais \\
\hline $\begin{array}{l}\text { C. } \\
\text { sphaerospermum } \\
\text { URM } 5350\end{array}$ & 128 & 16 & 64 & $+n$ & noterpeno testado apresentou valores de \\
\hline $\begin{array}{l}\text { C. } \\
\text { sphaerospermum } \\
\text { URM } 5455\end{array}$ & 256 & 16 & 16 & $+\mathrm{CI}$ & $\begin{array}{l}\text { I de } 128 \text { e } 256 \mu \mathrm{g} / \mathrm{mL} \text {. } \\
\text { De acordo com Aligianis et }\end{array}$ \\
\hline
\end{tabular}

Fonte: SANTOS, JMCG

*C - Controle de crescimento do micro-organismo em RPMI-1640, DMSO (0,5\%) e Tween 80 (2\%), sem monoterpeno ou antifúngicos.

\section{DISCUSSÃO}

Os terpenos são constituintes que fazem parte da composição dos óleos essenciais, que por sua vez são empregados na indústria na produção de perfumes e cosméticos, além de apresentarem efeitos farmacológicos ( EDRIS, 2007).

Diferentes testes têm sido utilizados para avaliar as propriedades farmacológicas das substâncias. Neste contexto, destacam-se os testes que utilizam os modelos in silico (expressão usada com o significado de “executado em computador"), que são rápidos, reprodutíveis e normalmente baseados em biorreguladores humanos, garantindo assim a segurança para a utilização do produto natural como um futuro fármaco (ANGELO, MAX, MARKUS et al., 2006; SRINIVAS et al., 2014). Neste estudo, a análise in silico do monoterpeno citral demonstrou várias promissoras atividades farmacológicas desta substância contra diferentes agentes infecciosos, em especial os fungos.

(2001) e Sartoratto et al. (2004), produtos com CIM até $500 \mu \mathrm{g} / \mathrm{mL}$ são considerados com forte poder antimicrobiano; produtos com CIM entre 600 e $1500 \mu \mathrm{g} / \mathrm{mL}$ moderado poder antimicrobiano, e produtos com CIM acima de $1500 \mu \mathrm{g} / \mathrm{mL}$ - fraco poder antimicrobiano. Desta forma, pode-se afirmar que o citral apresenta um forte efeito antifúngico contra as cepas de $C$. oxysporum e C. sphaerospermum.

Este potencial antifúngico in silico e in vitro do citral encontra-se de acordo com outros estudos que já demonstraram o potencial antimicrobiano deste composto contra outras espécies fúngicas, como por exemplo Penicillium digitatum (FISHER, PHILLIPS, 2008), Trichophyton mentagrophytes (PARK et al., 2009), Aspergillus flavus, Aspergillus fumigatus (LUO et al., 2004; MESA-ARANGO et al., 2009). 


\section{CONCLUSÕES}

Portanto, ao analisar os dados obtidos nesta pesquisa in silico e in vitro pode-se perceber que o monoterpeno citral apresenta uma promissora atividade antimicrobiana, em especial antifúngica contra espécies de $C$. oxysporum e $C$. sphaerospermum.

\section{REFERÊNCIAS}

Abramson CH, Aldama E, Sulbaran E. Exposure to citral, cinnamon and ruda disrupts the life cycle of a vector of Chagas disease. Am J Environ Sci, 3, 7 - 8, 2007.

Aligianis N, Kalpoutzakis E, Mitaku S, Chinou IB. Composition and antimicrobial activity of the essential oil of two Origanum species. J Agr Food Chem, 49(9), 41684170, 2001.

Angelo V, Max D, Markus AL. The Challenge of Predicting Drug Toxicity in silico. Bas Clin Phar Tox, 99, 195-208, 2006.

Belda-Galbis CM, Pina-Pérez MC, Leufvén A, Martínez A, Rodrigo D. Impact assessment of carvacrol and citral effect on Escherichia coli K12 and Listeria innocua growth. Food Control, 33, 536 - 544, 2013.

Chaouki W, Leger DY, Liagre B, Beneytout JL, Hmamouchi M. Citral inhibits cell proliferation and induces apoptosis and cell cycle arrest in MCF-7 cells. Fundam Clin

Pharmacol, 23, 549-556, 2009.

Cleeland R, Squires E. Evaluation of new antimicrobials in vitro and in experimental animal infection. In: Antibiotics in Laboratory Medicine. New York: Williams \& Wilkins, 1991. p. 739-787.

CLSI (Clinical and Laboratory Standards Institute), formely NCCLS (National
Committee for Clinical Laboratory

Standards). Reference method for broth dilution antifungal susceptibility testing of yeasts. Method M27-A2, $2^{\mathrm{a}}$ ed. Wayne Ed. 2002; 22: 1-29.

Correia RTM, Valente NYS, Criado PR, Martins JEC. Cromoblastomicose: relato de 27 casos e revisão da literatura. An Bras Dermatol, 85(4), 448-454, 2010.

De Hoog GS, Guarro J, Gené JL, Figueiras MJ. Atlas of Clinical Fungi, 2nd edn. Centraalbureau voor Schimmelcultures, Universitat Rovira i Virgilli, Utrecht/Reus, 2000. 1126 pp.

Dudai, N, Weinstein Y, Krup M, Rabinski T, Ofir R. Citral is a new inducer of caspase-3 in tumor cell lines. Planta Medica, 71, 484488, 2005.

Edris AE. Pharmaceutical and Therapeutic Potentials of Essential Oils and Their Individual Volatile Constituents: A Review. Phytother Res, 21(4), 308-323, 2007.

Eloff JN. A sensitive and quick microplate method to determine the minimal inhibitory concentration of plant extracts for bacteria. Planta Medica, 64(8), 711-713, 1998.

Farah IO, Trimble Q, Ndebele K, Mawson A. Retinods and citral modulated cell viability, metabolic stability, cell cycle progression and distribution in the A549 lung carcinoma cell line. Biomed Sci Instrum, 46, 410-21, 2010 .

Fisher K, Phillips C. Potential antimicrobial uses of essential oils in food: is citrus the answer? Trends Food Sci Technol, 19(3), $156-164,2008$.

Hadacek F, Greger H. Testing of antifungical natural products: methodologies, comparability of results and assay choise. Phytochem Anal, 11(3), 137-147, 2000.

Kwon-Chung KJ, Schwartz IS, Rybak BJ. A pulmonary fungus ball produced by 
Cladosporium cladosporioides. Am J Clin

Pathol, 64, 564-568, 1975.

Luo M, Jiang LK, Huang YX, Xiao M, Li B, Zou GL. Effects of citral on Aspergillus flavus spores by quasielastic light scattering and multiplex microanalysis techniques.

Acta Biochim Biophys Sin, 36, 277-283, 2004.

Mangprayool T, Kupittayanant S, Chudapongse N. Participation of citral in the bronchodilatory effect of ginger oil and possible mechanism of action. Fitoterapia, 89, 68-73, 2013.

Mesa-Arango AC, Montiel-Ramos J, Zapata B, Durán C, Betancur-Galvis L, Stashenko E. Citral and carvone chemotypes from the essential oils of Colombian Lippia alba (Mill.) N.E. Brown: composition, cytotoxicity and antifungal activity. Mem Inst Oswaldo Cruz, 104(6), 878, 2009.

Namratha N, Nadgir S, Kale M, Rathod R. Chromoblastomycosis due to Cladosporium carrionii. J Lab Physicians, 2(1), 47-48, 2010 .

Park MJ, Gwak KS, Yang I, Kim KW, Jeung EB, Chang JW, Choi IG. Effect of citral, eugenol, nerolidol and alpha-terpineol on the ultrastructural changes of Trichophyton mentagrophytes. Fitoterapia, 80, 290-296, 2009.

Pereira AA. Efeito inibitório de óleos essenciais sobre o crescimento de bactérias e fungos. 2006. 58 f. Dissertação (Mestrado em Ciência dos Alimentos). Universidade Federal de Lavras, Minas Gerais, 2006.

Poser GL, Mentz LA. Diversidade Biológica e Sistemas de Classificação. In: SIMÕES CMO, SCHENKEL EP, GOSMANN G. et al. Farmacognosia: da planta ao medicamento. 5. ed. Porto Alegre, RS: Ed. Da UFSC. 2004; Cap. 4: 75-89.

Sartoratto A, Machado ALM, Delarmelina C, Figueira, GM, Duarte, MCT, Rehder, VLG. Composition and antimicrobial activity of essential oils from aromatic plants used in Brazil. Braz. J. Microbiol, 35(4), 275 - 280, 2004.

Saddiq AA, Khayyat SA. Chemical and antimicrobial studies of monoterpene: Citral. Pestic Biochem PhysioL, 98, 89-93, 2010.

Schubert K. Morphotaxonomic revision of foliicolous Cladosporium species (hyphomycetes). Ph.D. dissertation. MartinLuther-University Halle-Wittenberg, Germany. http://sundoc.bibliothek.unihalle.de/diss-online/05/05H208/index.htm, 2005.

Simões CMO, Spitzer V. Óleos voláteis. In: Simões CMO, Schenkel EP, Gosmann G. et al. Farmacognosia: da planta ao medicamento. 5. ed. Porto Alegre, RS: Ed. Da UFSC. 2004; Cap. 18: 467-495.

Srinivas N, Sandeep KS, Anusha Y, Devendra BN. In Vitro Cytotoxic Evaluation and Detoxification of Monocrotaline (Mct) Alkaloid: An In Silico Approach. Int. Inv. J. Biochem. Bioinform, 2(3) 20-29, 2014.

Somolinos M, García D, Condón S, Mackey B, Pagán R. Inactivation of Escherichia coli by citral. J Appl Microbiol, 108, 1928$1939,2009$.

Xia H, Liang W, Song Q, Chen X, Hong J. The in vitro study of apoptosis in NB4 cell induced by citral. Cytotechnology, 65, 4957, 2013. 